\title{
Molecular imprinted polymer coated QCM for the detection of nandrolone
}

\author{
C.J.Percival $^{a}$, S.Stanley ${ }^{a}$, A.Braithwaite ${ }^{a}$, M.I.Newton ${ }^{*}{ }^{a}$ and G.McHale ${ }^{a}$ \\ a Department of Chemistry and Physics, The Nottingham Trent University, Clifton Lane, Nottingham NG11 8NS, \\ UK Fax: 44115 8486636; Tel: 44115 8483365; E-mail: Michael.Newton@ntu.ac.uk
}

\author{
This submission was created using the RSC Communication Template (DO NOT DELETE THIS TEXT) \\ (LINE INCLUDED FOR SPACING ONLY - DO NOT DELETE THIS TEXT)
}

\begin{abstract}
An acoustic wave sensor coated with an artificial biomimetic recognition element has been developed to selectively screen for nandrolone in the liquid phase. A highly specific covalently imprinted polymer (MIP) was spin coated on to one electrode of a quartz crystal microbalance (QCM) as a thin permeable film. Selective rebinding of the nandralone was observed as a frequency shift in the QCM for concentrations up to $0.2 \mathrm{ppm}$ with the sensor binding shown to favour nandrolone over analogous compounds.
\end{abstract}

\section{Introduction}

One of the most widely abused classes of banned drugs in sports is anabolic steroids (e.g. nandrolone). Anabolic steroids, which are related in structure and activity to the male hormone testosterone, are used by competitors to improve muscle strength and accelerate recovery times from exercise and so to extend their training effectiveness. The abuse of anabolic steroids in human and equine sports has led to a strict ban of anabolic steroids by national and international sports federations and by the Medical commission of the International Olympic Committee (IOC). Urine analysis is recommended for the detection of all banned drugs on the current IOC list ${ }^{1}$. A wide variety of methods for the detection of anabolic steroids in urine have been described. Immunoassay techniques can provide an effective means of screening numerous sample ${ }^{2,3}$ since they are rapid and inexpensive. However, these techniques have limited specificity and can give false positive results. Several TLC and HPTLC methods have been proposed ${ }^{4-7}$ but these methods have low sensitivity and the sample preparation is laborious. GC-MS has been proved to be a suitable technique for the detection of anabolic steroids ${ }^{8-12}$. In general however, GC-MS based procedures require expensive equipment and involve lengthy sample extraction and subsequent derivatisation.

Molecular recognition is a highly efficient and essential feature of biological systems in nature and as such has found application in biosensors ${ }^{13}$. Natural biological recognition systems, whilst highly selective, are of limited use, however, as a result of poor chemical and thermal stability, limited assay range, lifespan and expense. The use of artificial recognition materials has been proposed as a means of addressing some of the limitations inherent in biosensors. One of the most promising examples of artificially generated recognition materials are the molecularly imprinted polymers (MIP). MIPs are highly crosslinked polymers, which are inherently stable and capable of high selectivities approaching that of their natural counterparts. MIPs have become well established as a means of producing biomimetic recognition sites since first reported over 25 years ago ${ }^{14,15}$ with the selectivity and binding affinities of MIP being comparable to antibody-antigen interactions. Two distinct imprinting strategies have evolved: i) covalent and ii) noncovalent template polymerisations. Covalent imprinting involves the conversion of the template (or an analogue) into a polymerisable derivative that is then copolymerised with a suitable cross-linker to afford a resin that covalently incorporates the template. Covalent interactions between the template and the functional monomer have the advantage that the binding groups remain precisely orientated during elevated temperatures that are often a feature of the polymerisation process. The stability of the covalent bond produces a homogeneous population of binding sites within the imprinted polymer. In part this stability of the 'covalent complex' is responsible for the high binding affinities (in comparison to non covalent MIPs) associated with covalently imprinted polymers with in excess of $75 \%$ of print sites being reoccupied $^{16}$. Covalent imprinting has been widely used to produce MIPs that are selective for a range of analytes including, caffeine in human urine and serum ${ }^{17}$, the plant hormone indole acetic acid $^{18}$, macromolecules ${ }^{19}$, the chiral benzodiazepine ${ }^{20}$ and the steroid cholesterol ${ }^{21}$, which has formed the basis of our approach. In contrast, non-covalent imprinting involves the selfassembly of suitable functional monomers around the template molecule. Following addition of a cross-linker this assembly is then 'fixed' by polymerisation. Subsequent removal of the noncovalently bound template reveals vacant recognition sites that are specific to the target analyte in terms of their spatial and electronic environment. Non-covalent imprinting has inherent advantages over the covalent strategy as a consequence of the rapid and reversible nature of non-covalent interaction between the polymer and the template. Non-covalent imprinting has been reported with MIPs selective for many analytes including, Rand S-propranolol ${ }^{22}$, the odorant methylisoborneol ${ }^{23}$, steroids ${ }^{24}$, carboxylic acids ${ }^{25}$, nucleotide bases ${ }^{26}$, enantiomeric resolution of amino acids ${ }^{27,28}$ dyes $^{29}$ and $\mathrm{PAHs}{ }^{30}$. In this work we present an alternative technique for the selective direct detection of nandrolone in liquids using a covalently imprinted recognition element for potential use as a screening device.

\section{Experimental}

Reagents

All steroids and the functional monomer methacrylic acid (MAA) were purchased from Lancaster synthesis. The ethylene glycol dimethacrylate (EDMA), Phosgene and 4-Acetoxystyrene were supplied by Aldrich chemicals. The initiator 2,2'-Azobis(2methylpropionitrile) (AIBN) was supplied by Acros chemicals. All the solvents were of analytical grade and all reagents were used as supplied.

\section{Quartz Crystal Microbalance}

The quartz crystal microbalance consisted of a Maxtek PLO10 phased locked oscillator and an Agilent HP53132A universal counter interfaced to a microcomputer. The quartz crystals used were unpolished AT-cut, $25 \mathrm{~mm}$ diameter, with $\mathrm{Cr} / \mathrm{Au}$ contacts, operating at a fundamental resonant frequency of $5 \mathrm{MHz}$. The electrode area was approximately $133 \mathrm{~mm}^{2}$ (Maxtek model No. 149211-2) and the crystals were mounted in a Maxtek CHC-100 crystal holder. Prior to the application of the polymer coating to the electrode area, the crystals were prepared using Pirannha etch solution (1:3 $30 \% \mathrm{v} / \mathrm{v} \mathrm{H}_{2} \mathrm{O}_{2}$ : conc. $\left.\mathrm{H}_{2} \mathrm{SO}_{4}\right)$. The crystals were immersed in Pirannha etch solution for ten minutes, then rinsed with deionised water and ethanol and dried overnight.

\section{Molecular Imprinted Polymer Synthesis}

Prior to the formation of the imprinted polymer, nandrolone was first converted to a polymerisable derivative, in this case the 4vinylphenyl carbonate ester of nandrolone. The ester functions as a covalently bound template monomer that is easily cleaved to 
yield a non-covalent recognition site. The MIP film was produced via a four stage synthesis:

1 Preparation of 4-vinylphenol: 4vinyl phenol was prepared as per Corson et al. ${ }^{31}$ A mixture of $16.2 \mathrm{~g} .(0.10 \mathrm{~mole})$ of $p$ acetoxystyrene, $13.8 \mathrm{~g} .(0.25$ mole $)$ of potassium hydroxide and $140 \mathrm{ml}$. of water was stirred at $0^{\circ} \mathrm{C}$ until homogeneous $(2 \mathrm{hrs})$. The stirred cold solution was acidified to $p \mathrm{H} 8$ to produce $12 \mathrm{~g}$. (100\% yield) of 4-vinylphenol and the 4-vinylphenol product was identified by NMR.

2.Preparation of nandraolone chloroformate: $2.4 \mathrm{~g}$. $(8.3 \mathrm{mmol})$ of nandrolone in $30 \mathrm{ml}$ of dry THF with BHT (trace) was cooled on ice. Then $2.0 \mathrm{ml}$ of $\mathrm{NET}_{3}$ (dried over $\mathrm{KOH}$ ) was added dropwise to this cold solution. $9.60 \mathrm{ml}$ of phosgene dropwise to reduce fuming. This solution was stirred at $0^{\circ} \mathrm{C}$ under nitrogen for 6 hours.

3. Preparation of nandrolone (4-vinyl)phenyl carbonate: A solution containing $1.05 \mathrm{~g}(8.3 \mathrm{mmol})$ of 4-vinylphenol in $20 \mathrm{ml}$ of dry THF with BHT (trace) and 1.0ml.of $\mathrm{NET}_{3}$ (dried over $\mathrm{KOH}$ ) was added dropwise to the nandrolone chloroformate prepared in step two. The solution was stirred at room temperature overnight. The crude product was filtered and the solid discarded. The liquor was evaporated down with BHT. After evaporation, the residue was dissolved in DCM and washed with water. The organic layer was collected and evaporated down to yield product which was further cleaned by running through a silica column; the product 4-vinylphenyl carbonate ester of nandrolone was identified by NMR.

4. MIP synthesis: The monomer mixture consisting of the product 4-vinylphenyl carbonate ester of nandrolone $(1.5 \mathrm{mmol})$, Methacrylic acid $(6.0 \mathrm{mmmol})$, EDMA $(30 \mathrm{mmmol})$ and the porogenic solvent $(9: 1)$ hexane:tolune $(74 \mathrm{mmol})$, were dispensed into a sample tube and the initiator AIBN $(0.19 \mathrm{mmol})$ added the tube was the sealed and sonicated for 15 minutes. The polymerisation was then performed in a water bath at $65^{\circ} \mathrm{C}$ for 24 hours. The polymer was obtained as a brittle solid which, is crushed and sieved to $<38 \mu \mathrm{m}$. The ground polymer was extracted with methanol in a soxhlet apparatus overnight and then dried under vacuum at $40^{\circ} \mathrm{C}$.

The polymer was suspended in $1 \mathrm{M}$ sodium hydroxide in methanol and heated to refluxed for 6 hours. The cooled solutions are added to an excess of dilute $\mathrm{HCl}$. The products are filtered and washed with water, methanol and ether. Polymers were soxhlet extracted with methanol and then hexane and dried under vacuum at $40^{\circ} \mathrm{C}$. The films were then spin coated on to the crystals using the following technique: $10 \mathrm{mg}$.of PVC powder was dissolved in $5 \mathrm{ml}$ of THF and $30 \mathrm{mg}$ of MIP particles were suspended in solution with thorough stirring. A QCM was fixed via vacuum to a laboratory spin coater and coated with $10 \mu \mathrm{l}$ of the pvc/polymer solution and the suspension spread over the surface of the Au electrode by rotating at $600-900 \mathrm{rpm}$ for a period of five minutes.

\section{Evaluation of sensor response}

Solutions containing known amounts of the analyte were prepared in ethanol. The coated quartz crystals were placed into a beaker of ethanol until a stable response was obtained then, 6 $\mathrm{ml}$ of analyte solution were added to the stirred bulk ethanol solution via successive $1 \mathrm{ml}$ aliquots. The frequency was recorded until a stable response was obtained. After each $6 \mathrm{ml}$ addition of analyte the coated quartz crystal was soaked in the porogenic solvent for 10 minutes and the experiment was repeated. It is known that QCM are affected by changes in ambient temperature which cause a drift in resonant frequency. On the timescale of the experiment, the change in frequency caused by environmental conditions could be greater than that caused by the mass loading of the quartz crystal. In order to overcome this difficulty the frequencies of two quartz crystals immersed in the ethanol solution, one crystal coated with the MIP and one crystal coated with the non imprinted polymer, were measured simultaneously following the DQCM technique of Bruckenstein et. al. ${ }^{32}$. The resultant difference in frequency between the two crystals can be used to assess the response of the crystal to mass loading of the analyte as the non imprinted polymer displayed no change in resonant frequency with mass loading; all experiments were performed in this manner.

\section{Results and Discussion}

In figure 1 we show the change in resonant frequency of the QCM as a function of nandrolone concentration for two different coatings produced using an identical procedure. There is an initial linear decrease in frequency corresponding to increase in nandrolone concentration up to about $0.1 \mathrm{ppm}$ with a gradient of $-164 \pm 10 \mathrm{~Hz} \mathrm{ppm}^{-1}$, which reaches saturation at concentrations exceeding $0.2 \mathrm{ppm}$. Similar sorption isotherms have been observed by Haupt et al. ${ }^{22}$ for the binding of S-propranolol to a molecularly imprinted polymer and such sorption isotherms are expected for polymers that contain immobilised binding sites, such as found in MIPs. After the first cycle of exposures the response of the MIP degrades and gives a lower frequency change for the same analyte concentrations however, it can be seen that the response of fresh coatings is relativly reproducible. The affinity of the MIP towards nandrolone can be evaluated using a simple one site Langmuir-type binding isotherm anylsis $^{33} \mathrm{Kc}\left(\Delta \mathrm{f} / \Delta \mathrm{f}_{\infty}\right)+\left(\Delta \mathrm{f} / \Delta \mathrm{f}_{\infty}\right)=\mathrm{Kc}$ where $\mathrm{K}$ is the binding coefficient, $\mathrm{c}$ is the free analyte concentration at equilibrium $\Delta \mathrm{f}$ is the frequency change and $\Delta \mathrm{f}_{\infty}$ is the frequency corresponding to complete coverage. Although this does not reflect the real situation in the polymer it allows for a comparison of binding between polymers. Using the data shown, the calculated binding coefficient $\mathrm{K}=123.2 \pm 10.9 \mu \mathrm{M}^{-1}$ which is similar to those found in other MIPs ${ }^{34}$. In figure 1 we also show the response of the QCM to testosterone and epitestosterone; no significant change in resonant frequency was observed upon the addition of these steroids up to a concentration $2 \mathrm{ppm}$.

Whilst the stability of the MIP to repeated exposures of nandralone does not demonstrate reproducibility, we have shown that fresh MIP coated quartz crystal sensors does offer reasonable reproducibly. The natural extension of this work is to enhance the sensitivity of the sensors to enable a low cost screening technique of biological samples to be produced. This

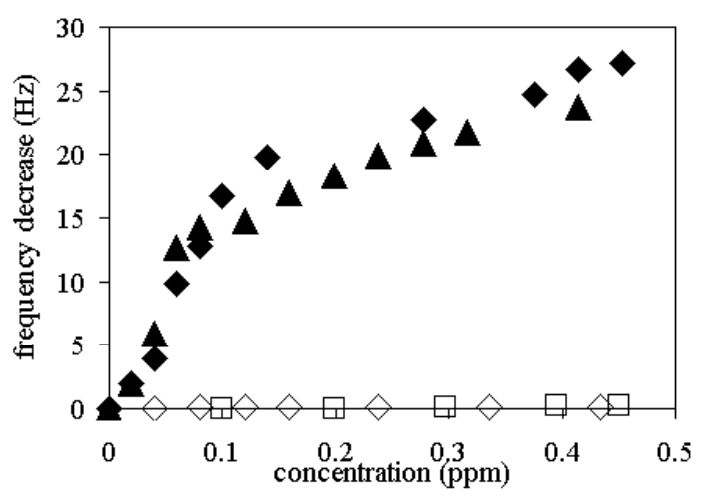

Fig1. Frequency decrease as a function of concentration for nandralone (solid triangles, solid diamonds), testosterone (open diamonds) and epitestostorone (open squares).

may be achieved firstly by increasing the concentration of binding sites in the MIP and secondly, by increasing the mass sensitivity of the acoustic device. The latter involves increasing the QCM resonant frequency; the upper limit for quartz crystal microbalances operating at the fundamental frequency being around $10 \mathrm{MHz}$. Alternatively, shear horizontal surface acoustic wave devices (SH-SAW) may be employed that will extend the range of operating frequencies by more than an order of magnitude ${ }^{23,35}$. Such acoustic wave devices may be incorporated 
in lightweight and low cost oscillator circuit allowing an inexpensive screening technique to be developed.

\section{References}

1. IOC. Olympic movement Anti-Doping Code. Lausanne: IOC, 2000.

2. H.H.D.Meyer and S.Hoffmann, Food Addit. Contam. 1987, 4, 149.

3. G.Degand, P.Schmitz, J.Maghuin-Rogister J. Chromatogr. 1989, 489, 235.

4. R.J.Verbeke, Chromatogr. 1979, 177, 69.

5. L.A.Van Ginkel, H.Van Blitterswijk, P.w.Zoontjes, D.Van Den Bosch, and R.W.Stephany, J. Chromatogr. 1988, 445, 385.

6. H.F.de Brabander,P.Vanhee, S.van Hoye, and R.Verbeke, J. Planar Chromatogr. 1989, 2, 33.

7. Th.Reuvers, Perogordo,E.Alimentaria, E. 1986, 170, 27.

8. E.Daeselerie, A.de Guesquierie and C. van Peteghem, J. Chromatogr. Sci. 1992, 30, 409.

9. A.Boenke, Anal. Chim. Acta. 1993, 275, 3 .

10. B.Le Bizec, M-P.Montrade, F.Monteau and F.Andre, Anal. Chim. Acta. 1993, 275, 123.

11. G.van Vyncht, P.Gaspar, E.Depauw and G.Maghuin-Rogister, $J$. Chromatogr. B. 1996, 686, 189.

12. Y.Galliard and G.J.Pepin, Chromatogr. A. 1997, 763, 149

13. R.S.Sethi, Biosens. Bioelectron. 1994, 9, 243

14. G.Wulff and A.Sarhan, Angew. Chem. Int. Ed. Engl. 1972, 11, 341.

15. G.Wulff, Angew. Chem. Int. Ed. Engl. 1995, 34, 1812

16. B.Sellergren, Anal. Chem. 1994, 66, 1578

17. C.Liang, H.Peng, X.Bao, L.Nie and S.Yao, Analyst 1999, 124, 1781

18. A.Kugimiya and T.Takeuchi, Electroanalysis 1999, 11, 1158

19. K.J.Shea, E.A.Thompson, S.D.Pandey and P.S.Beauchamp, J. Am Chem. Soc. 1980, 102, 3149

20. B.R.Hart, D.J.Rush and K.J. Shea, J. Am. Chem. Soc. 2000, 122, 460

21. M.J.Whitcombe, M.E.Rodriguez, P.Villar and E.N.Vulfson, J. Am. Chem. Soc. 1995, 117, 7105

22. K.Haupt, K.Noworyta and W.Kutner, Anal. Commun. 1999, 36, 391

23. H.S.Ji, S.McNiven, K.Ikebukuro and I.Karube, Anal. Chim. Acta 1999, 390, 93

24. O.Ramstrom, L.Ye, M. Krook and K. Mosbach, Anal. Commun 1998, 35, 9

25. O.Ramstrom, L.I.Andersson and K.Mosbach, J. Org. Chem. 1993, $\mathbf{5 8}, 7562$

26. D.A.Spivak, K.J. Shea, Macromoleculues 1998, 31, 2160

27. L.I.Andersson and K. Mosbach, J.Chromatogr. 1990, 516, 313

28. L.I.Andersson, D.J. O'Shanessy and K. Mosbach, J. Chromatogr. 1990, 513, 167

29. M.Glad, O.Norrlow, B.Sellergren, N.Siegbahn and K. Mosbach, $J$ Chromatogr. 1985, 347, 11

30. F.L.Dickert, M.Tortschanoff, W.E.Bulst and G. Fischerauer, Anal. Chem. 1999, 71, 4559

31. B.B.Corson, W.J.Heintzelman, L.H.Schwartzman, H.E.Tiefenthal, R.J. Lokken, J.E. Nickels, G.R.Atwood and F. Pavlik, J. J. Org. Chem. 1958, 23, 544

32. S.Bruckenstein, M. Michalski, A.Fensore, Z. Li and A.R.Hillman, Anal. Chem. 1994, 66, 1847-1852.

33. E.Yilmaz, K. Mosbach and K. Haupt, Anal. Commun. 1999, 36, 167 ,

34. G.Vlatakis, L.I.Andersson, R. Müller and K. Mosbach, Nature 1993, 364, 645

35. B.Jakoby, G.M.Ismail, M.P.Byfield and M.J.Vellekoop, Sens. Actuators A 1999, 76, 93 
